\title{
EHMTI-0181. Fast recovery of visual evoked potential amplitude after photostress in migraine patients between attacks
}

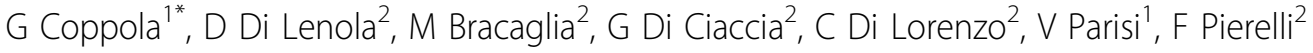 \\ From 4th European Headache and Migraine Trust International Congress: EHMTIC 2014 \\ Copenhagen, Denmark. 18-21 September 2014
}

\section{Background}

Subtle impaired macular vision was observed among different psychophysical experimental tasks in migraine patients.

\section{Aim}

Here we studied visual evoked potential (VEP) after photostress (PS) representing an objective index of the dynamic properties of macular performance after exposure to intense light stimulation.

\section{Method}

We recorded VEPs in basal condition and after PS in 43 migraine patients (19 with [MA] and 24 without [MO] aura) and 14 healthy volunteers (HV). PS is induced for a duration of 30 s by means of the bulb of a $200-\mathrm{W}$ lamp. We compared P100 implicit time and N75-P100 amplitude of baseline VEP with those collected every 20s up to 200s after PS.

\section{Results}

VEP parameters did not differed between groups at the baseline. In all groups, the VEPs recorded after PS showed a significant increase in latency at 20s. In HV, N75-P100 amplitude significantly decreased 20 s after PS, and recovered subsequently. There was no effect in the migraine groups. In fact, the percentage reduction in N75-P100 amplitude observed at 20s after photostress in MO and MA patients were lower than in HV (MO \& MA vs. HV $\mathrm{P}<0.05)$. When data of migraine patients were combined, the percentage of amplitude change at 20s was negatively correlated with number of days since the last migraine attack $(\mathrm{r}=-0.525, \mathrm{p}=0.02)$.

\section{Conclusion}

We documented altered recovery after PS under the influence of imminent attack. Whether or not present VEP findings are related to the ictal/interictal migraineur susceptibility to abnormal sensory perception, such as visual discomfort, remains to be determined.

No conflict of interest.

\section{Authors' details}

'Department of Neurophysiology of Vision and Neurophthalmology, G.B. Bietti Foundation-IRCCS, Roma, Italy. ${ }^{2}$ Department of medico-surgical sciences and biotechnologies, Sapienza University of Rome Polo Pontino, Latina, Italy.

Published: 18 September 2014

doi:10.1186/1129-2377-15-S1-E9

Cite this article as: Coppola et al: EHMTI-0181. Fast recovery of visual evoked potential amplitude after photostress in migraine patients between attacks. The Journal of Headache and Pain 2014 15(Suppl 1):E9. 